\title{
A NOTE ON GENERALIZED UNIQUE EXTENSION OF MEASURES*
}

\author{
DENG-YUAN HUANG
}

(Received 11 September 1970)

Communicated by J. B. Miller

In Theorem 1, we shall discuss some properties of semifinite measure, that is, the measure $\mu$ on a ring $R$ of sets with the property that, for every $E$ in $R$, $\mu(E)$ is equal to the least upper bound of $\mu(F)$ where $F$ runs over sets such that $F$ is in $R(F \subset E)$ and $\mu(F)<\infty$. Let $\sigma(R)$ be the $\sigma$-ring generated by $R$. To prove Theorem 2 we shall use the uniqueness theorem in Luther's paper [2], which is stated as a lemma in this paper. Theorem 2 is to the effect that for measures $\mu_{1}$ and $\mu_{2}$ on $\sigma(R), \mu_{1} \leqq \mu_{2}$ on $R$ implies $\mu_{1} \leqq \mu_{2}$ provided that $\overline{\mu_{i} / R}(i=1,2)$ is semifinite on $\sigma(R)$. Here $\overline{\mu_{i} / R}$ is the restriction, on $\sigma(R)$, of the outer measure $\left(\mu_{i} / R\right)^{*}$ induced by the restricted measure $\mu_{i} / R$ of $\mu_{i}$ on $R$. Definitions of terms are the same as [1] and [2].

Fix a set $X$. Let $R$ be a ring of subsets of $X$ and $\mu$ a measure on $R$. Let $\sigma(R)$ be the $\sigma$-ring generated by $R, \mu^{*}$ the outer measure induced by $\mu$ on the hereditary $\sigma$-ring $H(R)$ generated by $R$ and let $\bar{\mu}$ be the restriction of $\mu^{*}$ to $\sigma(R)$, that is, $\bar{\mu}=\mu^{*} / \sigma(R)$. Then $\bar{\mu}$ is a measure on $\sigma(R)$. In [2] Luther showed that semifiniteness of $\bar{\mu}$ implies that of $\mu$ on $R$ and that the semifiniteness of $\mu$ can not imply that of $\bar{\mu}$. We can prove the following:

THEOREM 1. If the measure $\mu$ is semifinite on $R$ and if for every $A \in \sigma(R)$ there is an $F$ in $R(F \subset A)$ such that $\bar{\mu}(A)=\mu(F)$ then $\bar{\mu}$ is semifinite.

Proof. For every $A$ in $\sigma(R)$, there is an $F$ in $R(F \subset A)$ such that

$$
\begin{aligned}
\tilde{\mu}(A) & =\mu(F) \\
& =\sup \{\mu(G): G \subset F, \mu(G)<\infty, G \in R\} \\
& \leqq \sup \{\bar{\mu}(G): G \subset A, \bar{\mu}(G)<\infty, G \in \sigma(R)\} \\
& \leqq \bar{\mu}(A) .
\end{aligned}
$$

Hence $\bar{\mu}$ is semifinite.

Remark. The converse of Theorem 1 is not true. For example, let $X=[0,1]$,

* It was supported by NSC of the Republic of China. 


$$
\begin{aligned}
R_{n}=\{A: A \text { is Lebesgue measurable and } A & \supset\left[0, \frac{1}{2}+\frac{1}{n+1}\right] \text { or } \\
A & \left.\subset\left(\frac{1}{2}+\frac{1}{n+1}, 1\right)\right\},
\end{aligned}
$$

( $n=1,2, \cdots$ ) and let $R=\bigcup_{1}^{\infty} R_{n}$. Then $R$ is a ring (actually is an algebra). Let $\mu$ be the Lebesgue measure restricted to $R$. Then $\left[0, \frac{1}{2}\right]$ is in $\sigma(R)$, so $F \subset\left[0, \frac{1}{2}\right], F$ in

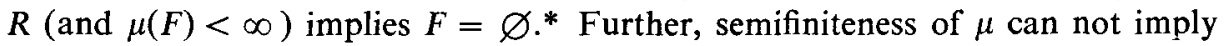
that $A$ in $\sigma(R)$ yields the existence of an $E$ in $R$ such that $\bar{\mu}(A)=\mu(E)$. Moreover, we can not get semifiniteness of $\mu$ even if also $A$ in $\sigma(R)$ implies the existence of an $F$ in $R(F \subset A)$ satisfying $\bar{\mu}(A)=\mu(F)$. For example, let $X$ be any infinite set and $R$ the ring of all finite subsets of $X$. Define $\mu$ on $R$ by

$$
\mu(E)= \begin{cases}0 & \text { if } E=\varnothing \\ \infty & \text { if } E \neq \varnothing\end{cases}
$$

The following lemma is due to Luther [2].

Lemma. Let $\mu$ be a measure on a ring $R$. If $\bar{\mu}$ is semifinite on $\sigma(R)$ then there exists a unique extension of $\mu$ to $\sigma(R)$.

By using this lemma we shall prove the following:

THEOREM 2. Let $\mu_{i}(i=1,2, \cdots)$ be measures on $\sigma(R)$. If $\overline{\mu_{i} / R}(i=1,2)$ is semifinite on $\sigma(R)$ and if $\mu_{1} \leqq \mu_{2}$ on $R$, then $\mu_{1} \leqq \mu_{2}$.

Proof. Let $M=\left\{E \in \sigma(R): \mu_{1}(E) \leqq \mu_{2}(E)\right\}$. Clearly, $M \supset R$. First we note that, if $\mu_{1}$ and $\mu_{2}$ are finite measures on $\sigma(R)$, then $\mu_{1} \leqq \mu_{2}$ on $\sigma(R)$. In fact, it is easy to see that $M$ is a monotone class. Hence $M \supset \sigma(R)$. This proves that

(i) for finite measures $\mu_{1}$ and $\mu_{2}, \mu_{1}(E) \leqq \mu_{2}(E)$ for all $E \in \dot{\sigma}(R)$.

Let $v_{i}=\mu_{t} / R(i=1,2)$. Then $\bar{v}_{i}$ is semifinite and $\mu_{i}=\bar{v}_{i}$ on $R$. By the lemma, we can obtain

(ii) $\bar{v}_{i}=\mu_{i}$ on $\sigma(R)$ for $i=1,2$.

Choose $E \in \sigma(R)$; in proving that $\mu_{1}(E) \leqq \mu_{2}(E)$, one may assume that $\mu_{2}(E)<\infty$. By semifiniteness of $\bar{v}_{1}$, we can find $F \in \sigma(R)(F \subset E)$ with $\bar{v}_{1}-\sigma$-finite measure such that $\bar{v}_{1}(E)=\bar{v}_{1}(F)$. Hence there is a sequence $\left\{F_{n}\right\}$ of sets in $R$ such that $F \subset \bigcup_{1}^{\infty} F_{n}$ and $v_{1}\left(F_{n}\right)<\infty$. Since by (ii)

$$
\bar{v}_{2}(F)=\mu_{2}(F) \leqq \mu_{2}(E)<\infty,
$$

there is a sequence $\left\{G_{n}\right\}$ of sets in $R$ such that $F \subset \bigcup_{1}^{\infty} G_{n}$ and $v_{2}\left(G_{n}\right)<\infty$. Hence we can suppose that

* I know this example from Dr. N. Y. Luther. 


$$
F \subset \bigcup_{1}^{\infty} H_{n}, H_{n} \in R, v_{i}\left(H_{n}\right)<\infty(i=1,2 ; n=1,2, \cdots) \text { and } H_{j} \cap H_{k}=\varnothing(j \neq k) \text {. }
$$

Therefore we see $F=\bigcup_{1}^{\infty}\left(H_{n} \cap F\right)$ and $\mu_{i}\left(H_{n}\right)<\infty$ and we get

$$
\begin{aligned}
\mu_{1}(F) & =\sum_{1}^{\infty} \mu_{1}\left(H_{n} \cap F\right)=\sum_{1}^{\infty}\left(\mu_{1}\right)_{H_{n}}(F) \leqq \sum_{1}^{\infty}\left(\mu_{2}\right)_{H_{n}}(F) \\
& =\sum_{1}^{\infty} \mu_{2}\left(H_{n} \cap F\right)=\mu_{2}(F) \leqq \mu_{2}(E),
\end{aligned}
$$

which leads to the required inequality,

$$
\mu_{1}(E)=\bar{v}_{1}(E)=\bar{v}_{1}(F)=\mu_{1}(F) \leqq \mu_{2}(E) . \quad \text { (by (ii)) }
$$

REMARK. If we drop the hypothesis that $\overrightarrow{\mu_{i} / R}$ is semifinite, then the result is false, even though $\bar{\mu}_{1}$ and $\bar{\mu}_{2}$ are semifinite or $\mu_{1}$ and $\mu_{2}$ are $\sigma$-finite, as the following example shows.

EXAMPLE. Let $R$ be a ring of subsets of a countable set $X$ with the property that every non-empty set in $R$ is infinite and such that $\sigma(R)$ is the class of all subsets of $X$. If, for every subset $E$ of $X, \mu_{1}(E)$ is the number of points in $E$ and $\mu_{2}(E)$ $=\frac{1}{2} \mu_{1}(E)$, then $\mu_{1}$ and $\mu_{2}$ are $\sigma$-finite on $\sigma(R)$ and $\overline{\mu_{1} / R}$ and $\overline{\mu_{2}} / R$ are not semifinite but $\bar{\mu}_{i}=\mu_{i}(i=1,2)$ is $\sigma$-finite (hence semifinite) on $\sigma(R)$. In this case $\mu_{1} \leqq \mu_{2}$ on $R$. but $\mu_{1} \geqq \mu_{2}$ and $\mu_{1} \neq \mu_{2}$ on $\sigma(R)$.

CoR. 1. Suppose $R$ is a ring, and $\mu_{1}$ and $\mu_{2}$ are measures on $\sigma(R)$ such that (i) $\mu_{1}(E) \leqq \mu_{2}(E)$ for all $E$ in $R$, and $(i i) \mu_{i} / R$ is $\sigma-$ finite. Then $\mu_{1} \leqq \mu_{2}$ on $\sigma(R)$.

Proof. Obviously, $\overrightarrow{\mu_{i} / R}$ is $\sigma$-finite and hence semifinite.

CoR. 2. Let $\mu_{i}(i=1,2)$ be measure on $\sigma(R)$. If $\mu_{i} / R(i=1,2)$ is semifinite and for every $A$ in $\sigma(R)$ there is an $F$ in $R(F \subset A)$ such that

$$
\overline{\mu_{i} / R}(A)=\mu_{i} / R(F)
$$

and if $\mu_{1} \leqq \mu_{2}$ on $R$, then $\mu_{1} \leqq \mu_{2}$.

Proof. By Theorem $1, \overline{\mu_{i}} / R(i=1,2)$ is semifinite, and by Theorem 2 , we get $\mu_{1} \leqq \mu_{2}$.

\section{References}

[1] S. K. Berberian, Measure and Integration, Macmillan (New York, 1965).

[2] N. Y. Luther, 'Unique Extension and Product Measures', Canad.J. Math. 19 (1967), 757-763.

Institute Of Mathematics

Academia Sinica

Republic Of China 